\title{
A genomewide ordered-subset linkage analysis for alcohol dependence in African-Americans
}

\author{
Shizhong Han ${ }^{1 *}$, Bao-Zhu Yang ${ }^{1,2}$, Henry R Kranzler ${ }^{3,4,5}$, Joel Gelernter ${ }^{1,2,6,7}$ \\ From Beyond the Genome: The true gene count, human evolution and disease genomics \\ Boston, MA, USA. 11-13 October 2010
}

\begin{abstract}
Alcohol dependence (AD) is extremely costly to individuals and to society. The etiology of AD is complex; genetic factors are known to be important to its development. With increasing evidence for the role of rare variants and copy number variation $(\mathrm{CNV})$ in complex diseases, linkage analysis remains a useful approach to gene discovery. An adequately powered linkage study can detect diverse genetic effects that segregate in families, including common variants, multiple rare variants within one locus and CNV.
\end{abstract}

Previously, we performed a genomewide linkage scan for AD in African- Americans (AA) (Gelernter 2009). The power of that linkage analysis could theoretically have been reduced by the presence of genetic heterogeneity owing to the variation of admixture proportions across families in AA. In the current study, we hypothesized that a sample whose genetic ancestry was more homogeneous would increase power to detect linkage. To test this hypothesis, we first completed a genomewide linkage scan with 6008 single nucleotide polymorphism markers to map loci linked with DSM-IV AD in 384 AA families, which were ascertained on the basis of multiple individuals affected with cocaine and/or opioid dependence. Using the admixture proportion as a covariate, we then applied the ordered subset linkage analysis (OSA) technique to identify a more homogeneous subset of families to determine whether it increased evidence for linkage with AD. Statistically significant increases in Kong-Cox LOD scores were observed on chromosomes $4(P=0.0001), 12(P=0.021)$, $15(P=0.026)$ and $22(P=0.0069)$. In a subset of $44 \mathrm{AA}$ families (with African ancestry proportions ranging from 0.858 to0.996), we observed a genomewide significant linkage at $180 \mathrm{cM}$ on chromosome 4 (Kong-Cox
$\mathrm{LOD}=4.1$, pointwise $\mathrm{P}=0.00001$, empirical genomewide $\mathrm{P}=0.008)$. GLRA3, the gene encoding a subunit of the glycine neurotransmitter receptor, is located in this peak region; thus, it deserves further research attention. A parametric linkage analysis confirmed the significant findings from the non-parametric linkage analysis. Our results show new evidence for linkage and demonstrate that the admixture proportion can be used effectively as a covariate to reduce genetic heterogeneity and enhance the detection of linkage signals in AA using the OSA technique. The current study provides important clues for candidate gene and deep sequencing studies to identity causal variants for AD.

\section{Author details}

'Department of Psychiatry, Division of Human Genetics, Yale University School of Medicine, New Haven, CT 06511, USA. ${ }^{2}$ VA CT Healthcare Center 116A2; 950 Campbell Avenue; West Haven, CT 06516, USA. ${ }^{3}$ Department of Psychiatry, University of Connecticut School of Medicine, Farmington, CT 06030, USA. ${ }^{4}$ Department of Genetics, University of Connecticut School of Medicine, Farmington, CT 06030, USA. ${ }^{5}$ Department of Developmental Biology, University of Connecticut School of Medicine, Farmington, CT 06030, USA. ${ }^{6}$ Department of Genetics, Yale University School of Medicine, New Haven, CT 06511, USA. Department of Neurobiology, Yale University School of Medicine, New Haven, CT 06511, USA.

Published: 11 October 2010

doi:10.1186/gb-2010-11-S1-O6

Cite this article as: Han et al: A genomewide ordered-subset linkage analysis for alcohol dependence in African-Americans. Genome Biology 2010 11(Suppl 1):06. 